\title{
The Effect of Antibiotics on the Respiration of Microorganism in Northern Ohio Rivers
}

\author{
David Patrick Gonia ${ }^{\mathrm{a}}$, Patricia Talarczyk ${ }^{\mathrm{a}}$
}

This experiment was conducted for the purpose of inquiring how the concentration of antibiotics, specifically Amoxicillin and Cephalexin, would affect the productivity of aquatic microorganisms within Northeastern Ohio rivers. In acknowledgment of the growing concerns over agricultural pollution, the study was devised to increase current knowledge of how the presence of antibiotics could affect aquatic microorganisms other the well-documented creation of antibiotic-resistant bacteria. In order to achieve the research goal, water was collected from a Northeastern Ohio river and then tested with various amounts of antibiotics. Over the following five days, the concentration of dissolved oxygen was measured which was used to calculate the overall rate of respiration of the sample. The overall respiration rates of the samples with Amoxicillin were $0.0369 \mathrm{mg} / \mathrm{L} \cdot \mathrm{hr}$ (milligram per liter per hour) with no antibiotics, $0.835 \mathrm{mg} / \mathrm{L} \cdot \mathrm{hr}$ with $250 \mathrm{mg}, 1.16 \mathrm{mg} / \mathrm{L} \cdot \mathrm{hr}$ with $500 \mathrm{mg}$, and $0.951 \mathrm{mg} / \mathrm{L} \cdot \mathrm{hr}$ with $750 \mathrm{mg}$. The samples with Cephalexin yielded rates of $0.963 \mathrm{mg} / \mathrm{L} \cdot \mathrm{hr}$ with $250 \mathrm{mg}, 0 . \mathrm{mg} / \mathrm{L} \cdot \mathrm{hr}$ with $500 \mathrm{mg}, \mathrm{mg} / \mathrm{L} \cdot \mathrm{hr}$ with $750 \mathrm{mg}$. These yielded Rvalues of 0.694 and 0.788 respectively. This means that the null hypothesis was rejected, thus showing a statistically significant correlation. However, the alternate hypothesis was not supported as a positive correlation between the respiration rate and antibiotic concentration was shown.

Keywords: Antibiotics; Pollution; Respiration Rate

Water is a fundamental need of organisms, especially humans. However, not all water is potable. Humans require fresh water in order to live and form their communities around this vital resource. From rivers to lakes, humans build their communities around sources of fresh water to supply their population with this necessity. These water sources are exuberantly valuable and none so as much as the Great Lakes, the largest freshwater bodies on the planet, and those rivers that flow into them. Yet recently, with increasing levels of agriculture production causing rising water pollution, the issues of preventing the contamination of the water supply are becoming more pressing. Agriculture pollution consists of several chemicals, the most significant of which being fertilizers and antibiotics. These pollutants have major impacts on the environment as well as human health. Both pollutants enter river systems as runoff where they eventually flow into larger bodies. Fertilizers are by far the most studied of the pollutants, with countless papers looking into their effects on algae growth, particularly how an increased presence of antibiotics yield the formation of algal blooms (Molot, 2017). In addition, studies have also focused on possible steps to limit their impact through mens such as government regulations (Pick, 2016). In contrast, fewer studies have been done on antibiotics, meaning that much knowledge could be gained from analyzing their effects on the environment.

Antibiotics have been used for the treatment of bacterial infections in various different fields. One of the most prominent uses is agriculture in which around 80 percent of antibiotics sold in the US are used primarily to promote growth and to prevent infection (Ventola, 2015). The issues with this practice stem from the overuse of these antibiotics with an around 100,000-1,000,000 tons of antibiotics globally (Zhang et al., 2014). This is made worse due to the animals' inefficient absorption of these antibiotics with 40-90 percent of the antibiotics excreted by the animal (Zhang et al., 2014). The antibiotics are released as waste and thus can runoff off into rivers and streams, polluting them. However, not all of the antibiotics the animals excrete act as pollutants:between 20-99 percent of the antibiotics are removed from animal waste before they can enter aquatic environments (Zhang et al., 2014). Even with the removal of a portion of the antibiotics, there is such great variation in the percentage of antibiotics that are removed that serious concerns are raised about the amount of antibiotic being released into nearby ecosystems. Such high concentrations leads to major worries about the impacts antibiotics may have on humans and the environment.

The most common concern with antibiotics in the water system revolves around the creation of antibiotic-resistant bacteria. Throughout many different fields of study, these resistant bacteria have been found to provide great danger to humans, who rely on those antibiotics to cure infections of those very same bacteria. However, the focus of antibiotic-related research has remained largely on bacteria found within the water and their growing resistance instead of on the other microorganisms inside the water, mainly algae. This study addresses the possible environmental impacts of antibiotics on the rivers flowing into Lake Erie, specifically in the area of Northeastern Ohio, by looking at some of the major river inputs of the lake and determining the effects of antibiotics on the population of the microorganisms. The aim is to answer the question, how does antibiotic concentration affect the respiration rate of a sample of river water?

\section{Literature Review}

Antibiotic is the general term given to the various chemicals that have the ability to lower the number of bacteria within a population. However, antibiotics fundamentally function in different ways to achieve the same result. Antibiotics can be either bacteriostatic, meaning they inhibit cell growth, or bactericidal, meaning they induce cell death (Kohanski, Dwyer, and Collins, 2010). Currently, most antibiotics are bactericidal, achieving their purposes through multiple different methods including inhibiting DNA synthesis, RNA synthesis, cell wall synthesis, or protein synthesis (Kohanski, Dwyer, and Collins, 2010). The antibiotics used within this study are bactericidal and specifically inhibit cell wall synthesis. They achieve this by preventing the bonds from 
forming in the peptidoglycan cell wall that makes up certain bacterium (Kohanski, Dwyer, and Collins, 2010). This type of cell wall differs from those of plants which consist of cellulose, but the knowledge of how these antibiotics work is fundamental to understanding the environmental effects of antibiotics as it must be recognized that different antibiotics may work in significantly different ways even if they achieve the same goal. This provides context for the different antibiotics used in other studies and allows comparisons to be create. Moreover, the knowledge of how antibiotics affect organisms on a cellular level may provide insights into how antibiotics might affect organisms such as microscopic algae that have certain similarities and differences within their biochemistry.

Regardless of their specific type, antibiotics have been shown to be present in many different river systems around the world. This creates both environmental and health concerns as antibiotics at this high concentration have shown to cause issues. As far back as 1999, fourteen different antibiotics were found in high enough concentrations to lead to antibiotic resistance with bacteria in US waterways (Kolpin et al., 2002). Furthermore, a study found bacteria such as E. coli, Salmonella, Shigella, and Streptococcus sp. present in high concentration within water sources in Nigeria that were resistant to twelve separate antibiotics, including Penicillins and Cephalosporins (Onuoha, 2017). Additionally, a total of 88 percent of E. coli isolated from rivers north of Beijing were resistant to one or more types of antibiotics (Zhang et al., 2014). The concentrations of antibiotics in the water supply caused by their overuse in agriculture has shown to be in high enough concentrations to create resistant bacteria. From North America to Africa to Asia, the risk of antibiotic pollution and the effects it has on the environment is well documented and shows the prevalence of the issue worldwide. This poses major concerns as these resistant bacteria contaminate the water supply and cause infections. The prevalence of these resistant bacteria requires examinations of water sources around the world to further identify how problematic the concentration of antibiotics is.

Environmentally, antibiotics have been shown to have a negative impact on microbiological ecosystems as a whole. Specifically, the presence of antibiotics has been found to cause a reduction in the both the overall microbial biodiversity and the growth of those same organism, not just that of bacteria that is supposed to be targeted (Grenni, Ancona, and Caracciolo, 2018). Moreover, antibiotics can functionally destabilize microbial ecosystems by lowering the growth and enzyme activity of microbial communities and ultimately changing their ecological functions (Bashir and Cho, 2016). Khawaja Muhammad Imran Bashir and Man-Gi Cho of Dongseo University support this idea with their study that identified a significant reduction in the growth of nearly 100 percent at some concentration in strains of chlorophyte algae in the presence of the antibiotics tetracycline and kanamycin (Bashir and Cho, 2016). The unintended effects of antibiotics are thus shown as microorganisms other than bacteria are identified as having their growth inhibited by antibiotics. While the antibiotics discussed differ from those used in this study as they work to inhibit protein synthesis rather than the cell wall, the effects of certain antibiotics has been identified and suggest the overall harmful effects of antibiotics on organisms besides bacteria (Kohanski, Dwyer, and Collins, 2010). With the known issues with the concentrations of antibiotics found within water systems, the danger on the ecosystem has been well identified. Antibiotics can not only harm bacteria, their intended targets, but other microorganisms as well, which means that antibiotics have the potential to be a significant threat to the environment as a whole, not just to human health.

The hazards of antibiotics in the water system presented in the studies both from resistant bacteria risk to humans and the microbial ecosystem health call for further research. While these issues have been studied before, most research has remained focused on specific microorganisms instead of a larger community. Moreover, how antibiotics affect them on a macro scale. Furthermore, no studies have specifically been done to look at the effects of antibiotics on the Northeastern Ohio water system. Increased knowledge of how antibiotics affect the microorganisms that are in the Lake Erie watershed will help to further develop an understanding of antibiotics and their effects on ecosystems.

\section{Hypothesis}

Basic conceptual knowledge of antibiotics states that they lower the number of microorganisms. With this knowledge, one can ascertain that including antibiotics would cause respiration rates to lower as there are fewer microorganisms to photosynthesize. An alternate hypothesis was then formulated that if the concentration of antibiotics is increased within the environment, then the respiration rate of the microorganisms would then decrease.

In addition, a null hypothesis was required to test the data for the statistical significance, which would consist of a Pears on correlation. The null hypothesis was of the overall thinking that there would be no significant effect on the respiration rate of the microorganisms with the addition of antibiotics. This would provide a suitable test to analyze the results of the collected data.

\section{Method}

This study utilized a scientific experimental method. Samples of water were collected from tributaries of Lake Erie across Northern Ohio to be treated with the antibiotics Cephalexin and Amoxicillin. Both of these antibiotics are bactericidal. More specifically these antibiotics induce cell death through disrupting cell wall synthesis of the organisms. Seven test groups were established with all having varying concentrations of antibiotics of the two types. Over a period of five days, a dissolved oxygen probe was utilized to determine the dissolved oxygen levels of the organisms within the sample. Combined with the change in time, the respiration rate of the sample was able to be determined which provides data on how the organisms were affected by the antibiotics.

The experimental method was the most applicable to the study due to its ability to produce quantitative data. This was fundamental in effectively determining the true effect that antibiotics would have on the sample. In order to accurately establish a relationship between the respiration rate of an organism and the presence of antibiotics within the environment, quantitative data was the most efficient method of generating any correlation. The data could be used to establish statistical significance. In contrast, 
using qualitative methods of research would have yielded inconclusive data as the appearance of the samples could not yield solid enough information on how the organisms were affected due to their microscopic size.

From previous experiences in biology courses and the work done in previous research, this method was able to be developed. Labs done within biology classes showed how dissolved oxygen probes can be used effectively in determining the respiration rate of organisms. This allowed for accurate determinations of the relative number of individuals within the populations as the higher the respiration, the more organisms that are living. Thus, if the antibiotics were to cause changes in the sample, the rate of respiration would thus be altered as well. However, I did come across other methods of testing the water, but most of these included the use of tools such as centrifuges that were not able to be obtained for this study, so these methods, while maybe more accurate were not used.

Rivers were chosen due to their size with each no shorter than 40 miles in length as well as their capability to be accessed as not all rivers were suitably close or in conditions that made sample collection possible. Testing multiple rivers allowed for a larger sample size that could aid in determining whether the results were just a singular case or showed an overall trend in the effects of antibiotics.

In order to access the rivers, public parks were utilized as they provided free public access to the water. Samples were then taken along banks and as far down from the surface as possible in order to obtain the most biologically varied sample as possible. This was repeated until seven containers of $32 \mathrm{oz}$ in volume were filled with samples. At this point, the samples were transported back to the testing location. Due to a shortage of containers for the samples, samples from each river were collected consecutively with no overlap. This required each test to be carried out roughly a week apart from each other. This was not the ideal testing method but was necessary due to the restraints of the resources.

The antibiotics Cephalexin and Amoxicillin were chosen due to their prevalence as well as their function of inhibiting cell wall synthesis ${ }^{5}$. This meant that they could be easily obtained for the experiment as they are two of the most common antibiotics sold. For this experiment, the antibiotics were purchased from a pet supply store and were selected due to their lower price. Additionally, Cephalexin and Amoxicillin provide a baseline for how many other antibiotics would affect the microorganisms within a river due to their function as cell wall synthesis inhibitors. Therefore, data collected in this study could then be applied on a broader scale to identify how large-scale pollution of antibiotics would affect an entire ecosystem.

For this experiment, each sample was tested using various concentrations of antibiotic. $250 \mathrm{mg}, 500 \mathrm{mg}$, and $750 \mathrm{mg}$ were chosen due to their significantly higher concentrations than those that are usually found in rivers ${ }^{6}$. Additionally, these concentrations were easily obtained as the antibiotics purchased came in increments of $250 \mathrm{mg}$. The effects of antibiotics on the organisms could then be clearly shown due to the high concentrations that would persist in each sample that would yield results if any relationship between respiration rate and antibiotic presence exists. In order to achieve the masses of the antibiotics that needed to be added, a $250 \mathrm{mg}$ pill was chopped up and then massed to achieve the necessary amounts for the experiment. At this point, the antibiotics were then dissolved straight into the sample containers.

The respiration rate utilized seven test groups to gather sufficient data. One control group existed which contained no antibiotics within it. This sample allowed for a comparison of the respiration rates of the other samples that had antibiotics added. The next six groups contained the two different types of antibiotics at their three different concentrations. This would thus show an overall trend in how the antibiotics would affect the respiration rate of the entire sample and thus, how the antibiotics affected the overall population as lower respiration rates would mean fewer organisms in the sample.

The seven samples were stored in complete darkness to guarantee no photosynthesis could occur and cause any unintended increases to dissolved oxygen levels. Each day, beginning with the day the antibiotics were first put into the samples, a dissolved oxygen probe was inserted into each sample to determine the dissolved oxygen concentrations. For five days, this was repeated at this length of time was long enough for the changes to the concentration brought about by the antibiotics' presence to occur if they were to exist.

During the experiment, some variables were standardized in order to increase the experiment's accuracy. The light level remained constant for the seven samples that required light. If different light levels were experienced by samples then the rate of respiration may be skewed towards those samples that received more light. Additionally, the temperature was maintained at close to $20^{\circ} \mathrm{C}$ to make sure a change in temperature that may cause an unwanted change in the rate did not occur.

\section{Results}

The results of the experiment yielded the data shown in Table 1. The table contains both the values for the samples with Amoxicillin and Cephalexin along with their increasing concentration and the dissolved oxygen concentrations. The left column contains the concentrations of antibiotics in milligrams along with which antibiotic was used. The dissolved oxygen is then in the next columns. The date was collected is located underneath the dissolved oxygen header and states that the day the data was collected along with the time. All data showed a general decrease in dissolved oxygen as organisms within the samples only used the oxygen due to respiration because no photosynthesis could occur in the absence of light. 
Table 1

Relationship Between Antibiotic Concentration and Dissol ved Oxygen

\begin{tabular}{|c|c|c|c|c|c|}
\hline \multirow[b]{2}{*}{$\begin{array}{l}\text { Antibiotic } \\
\text { Concentration }\end{array}$} & \multicolumn{5}{|c|}{ Dissolved Oxygen Concentration $(\mathrm{mg} / \mathrm{L})$} \\
\hline & $2 / 27 / 1918: 30$ & $2 / 28 / 1921: 30$ & $3 / 1 / 196: 00$ & $3 / 2 / 1923: 30$ & $3 / 3 / 1918: 00$ \\
\hline \multicolumn{6}{|l|}{ Amoxicillin } \\
\hline 0 (mg) & 12.43 & 11.38 & 10.82 & 9.27 & 9.01 \\
\hline $250(\mathrm{mg})$ & 12.97 & 11.87 & 11.49 & 10.10 & 9.74 \\
\hline $500(\mathrm{mg})$ & 13.11 & 11.70 & 11.24 & 9.29 & 9.06 \\
\hline $750(\mathrm{mg})$ & 13.09 & 11.59 & 11.12 & 8.80 & 8.41 \\
\hline \multicolumn{6}{|l|}{ Cephalexin } \\
\hline 0 (mg) & 12.43 & 11.38 & 10.82 & 9.27 & 9.01 \\
\hline $250(\mathrm{mg})$ & 13.00 & 11.83 & 11.31 & 10.03 & 9.68 \\
\hline $500(\mathrm{mg})$ & 13.14 & 11.65 & 11.28 & 9.62 & 9.18 \\
\hline 750 (mg) & 13.18 & 11.52 & 11.03 & 9.15 & 8.68 \\
\hline
\end{tabular}

Since the dissolved oxygen was only being used in respiration, the rate at which the collective sample was respiring was calculated. Table 2 shows the respiration rates of the samples at each concentration. This was calculated by graphing the time of each sample being collected against the dissolved oxygen concentrations. The time was converted into hours with the first time, $2 / 27 / 1918: 30$, being made 0 . The average rate of change was then determined and used as the respiration rates in Table 2 . Due to this the data was measured in milligram per liter per hour $(\mathrm{mg} / \mathrm{L} \cdot \mathrm{hr})$.

Table 2

Relationship Between Antibiotic Concentration and Respiration Rate

\begin{tabular}{cl}
\hline Antibiotic Concentration & Respiration Rate \\
\hline Amoxicillin & \\
$0(\mathrm{mg})$ & $0.0369 \mathrm{mg} / \mathrm{L} \cdot \mathrm{hr}$ \\
$250(\mathrm{mg})$ & $0.0340 \mathrm{mg} / \mathrm{L} \cdot \mathrm{hr}$ \\
$500(\mathrm{mg})$ & $0.0436 \mathrm{mg} / \mathrm{L} \cdot \mathrm{hr}$ \\
$750(\mathrm{mg})$ & $0.0506 \mathrm{mg} / \mathrm{L} \cdot \mathrm{hr}$ \\
Cephal exin & \\
$0(\mathrm{mg})$ & $0.0369 \mathrm{mg} / \mathrm{L} \cdot \mathrm{hr}$ \\
$250(\mathrm{mg})$ & $0.0346 \mathrm{mg} / \mathrm{L} \cdot \mathrm{hr}$ \\
$500(\mathrm{mg})$ & $0.0411 \mathrm{mg} / \mathrm{L} \cdot \mathrm{hr}$ \\
$750(\mathrm{mg})$ & $0.0469 \mathrm{mg} / \mathrm{L} \cdot \mathrm{hr}$ \\
\hline
\end{tabular}

A graph was then created in order to illustrate how the respiration rate contained a general trend of increase as the concentration of antibiotics increased. Figure 1 contains two lines: the red line is the respiration rates of the samples with Amoxicillin while the blue line is the respiration rates of the samples with Cephalexin. This was done to easily compare the results of both antibiotics to identify if their results supported each other. 
Relationship Between Antbiotic Concentration and Respiration Rate

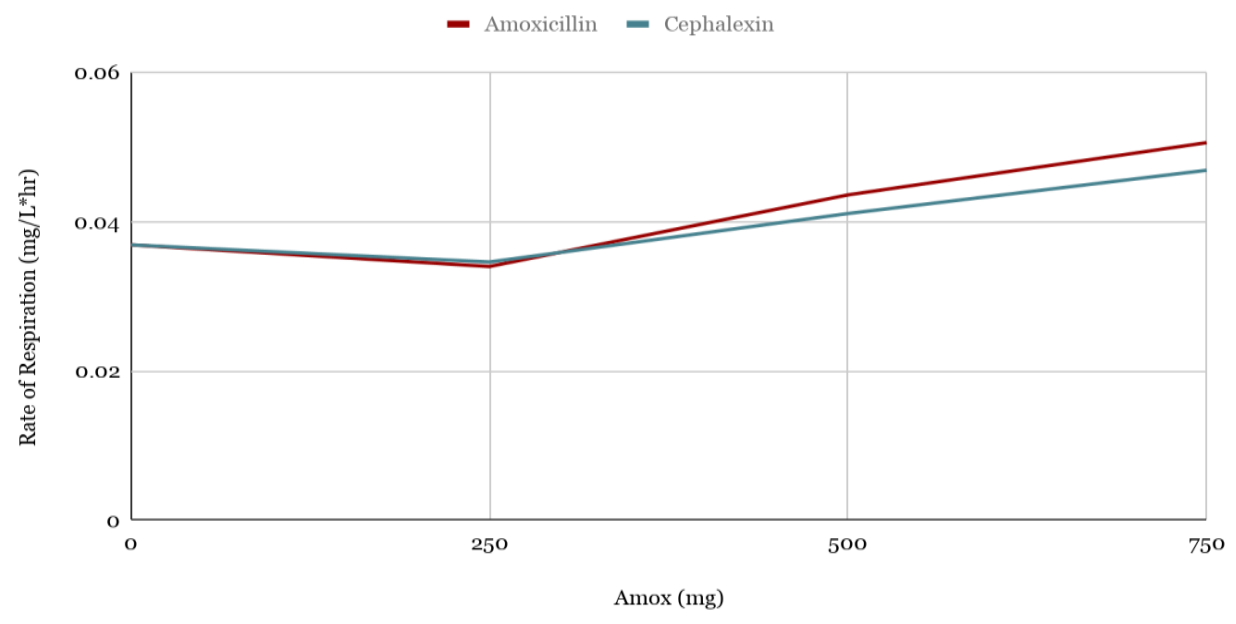

Figure 1 (Relationship Between Antibiotics and Respiration Rate)

\section{Analysis}

As shown by the results for both Amoxicillin and Cephalexin, as the concentration of antibiotics increased, the respiration rates generally increased. The respiration rate of the sample with no antibiotics was $0.0369 \mathrm{mg} / \mathrm{L} \cdot \mathrm{hr}$. For both the Amoxicillin and Cephalexin, the respiration rate decreased with $250 \mathrm{mg}$ of antibiotic to $0.0340 \mathrm{mg} / \mathrm{L} \cdot \mathrm{hr}$ and $0.0346 \mathrm{mg} / \mathrm{L} \cdot \mathrm{hr}$ respectively. At this point, the results indicate an increase in respiration rate with the increasing Amoxicillin concentrations as at $500 \mathrm{mg}$, the respiration rate was $0.0436 \mathrm{mg} / \mathrm{L} \cdot \mathrm{hr}$ and at $750 \mathrm{mg}$ the respiration $0.0506 \mathrm{mg} / \mathrm{L} \cdot \mathrm{hr}$. This trend is corroborated by the data from the samples of Cephalexin as at $500 \mathrm{mg}$ the respiration rate was $0.0411 \mathrm{mg} / \mathrm{L} \cdot \mathrm{hr}$ and at $750 \mathrm{mg}$ the rate was $0.0469 \mathrm{mg} / \mathrm{L} \cdot \mathrm{hr}$. For both antibiotics, the results indicate an initial decrease in the respiration at $250 \mathrm{mg}$ of antibiotic within the samples and then an increase to the respiration rate at the increasing antibiotic concentration beyond this value. Regardless of the dip in respiration rate, the data indicates an overall increase in respiration rate as antibiotic concentration increased. However, a statistical analysis must be performed to determine if the data is statistically significant and thus show any relationship between the antibiotic concentration and the respiration rate of the samples.

The null hypothesis for this study stated that there would be no statistical significance between the concentration of antibiotics and the respiration rate. In order to evaluate this hypothesis, two Pearson correlations were performed. This was chosen as the Pearson test is used to identify a correlation between two variables, like those of antibiotic concentration and respiration rate used within this study. For the statistical significance to be tested properly using this method, an R-value must be determined for both the samples with Amoxicillin and those with Cephalexin. To achieve this, a Pearson correlation calculator was used to determine the R-values for the data (Calculate Correlation Co-efficient, n.d.). The values returned as 0.884 for the samples with Amoxicillin and 0.872 for the samples with Cephalexin. In order for the data to be determine as significant, the R-values were compared to a critical value in which they had be greater than to show a statistically significant correlation. The critical value at 0.05 ( $95 \%$ significance) was gained from a chart of values and determined to be 0.729 . Since both the R-values exceeded this number, the data was shown as statistical significance. The data is therefore shown to be correlated and not simply occurring by random chance. Due to this, the null hypothesis was rejected. However, the data did not support the alternate hypothesis as a positive correlation between the concentration of antibiotics and the respiration rate was established by the data.. In contrast, the alternate hy pothesis stated a negative correlation would exist between the variables. Regardless, the data shows clearly that as antibiotic concentration increases, the respiration rate increases.

With regards to the results, a few limitations existed in the study that could have potentially affected the data. The most significant limitation with this study was the time at which it was conducted in the winter. In Northeastern Ohio, where the study was conducted, weather in the winter can become cold and unpredictable, limiting the access to the water and making collecting the samples difficult. This resulted in few trials being able to be performed, meaning that little data was collected, making this study very small in scale. Additionally, the winter months also meant lower quantities of organisms within the water and thus results that may not demonstrate the effects of the antibiotics in a more biologically diverse sample as it is well known that microorganisms are less productive in colder temperature.

\section{Conclusion}

While the results did not support the initial alternate hypothesis, they remained nonetheless statistically significant. The findings of this study indicate that the respiration rate of a sample increases with the concentration of the antibiotics within and remained similar for both Amoxicillin and Cephalexin. This suggests that if the concentration of antibiotics were high enough in a body of water, they could actually promote overall growth and respiration within the environment. This seems to contradict the 
majority of studies currently published as most suggest that antibiotics by their very nature should work to kill microorganisms and thus lower the respiration rate.

The study and its hypotheses followed along with the current body of knowledge and its findings on the effects that antibiotics have on organisms. Specifically, the assumption was made that antibiotics would work to kill most if not all microorganisms within the sample due to the major portion of them that would likely have cell walls that the antibiotics could work to harm in order to kill them. Regardless of their created purpose, studies indicate antibiotics as harmful to growth of different microorganisms. As stated previously, the study conducted by Bashir and Cho illustrated that the presence of antibiotics reduced the growth of two different species of eukaryotic algae (Bashir and Cho, 2016). Additionally, the findings of Paola Grenni, Valeria Ancona, and Anna Barra Caracciolo of the Water Research Institute further seem to oppose my own discoveries as they discuss the reduction of growth of microorganism and loss of biodiversity within an ecosystem due to the presence of antibiotics (Grenni, Ancona, and Caracciolo, 2018). In turn, my results appear to directly oppose the findings of the current body of work; however, these very same studies that contradict my findings can actually provide an explanation of the results that situate it firmly within the body of knowledge. Rather than the antibiotics harming all organisms within the sample like initially thought, it is possible that the antibiotics used in this study, which specifically inhibited the synthesis of the cell walls of certain microorganism, only killed specific organisms within the sample. In turn, due to the death of only part of the organisms within the sample, the resources that they had used along with the space that they had taken up would be open for the other organisms that were unaffected to increase their growth and thus respire more at higher concentrations. Meanwhile, at the lower concentration, there could have possibly been enough of the targeted bacteria left to prevent the rapid growth of the other organisms, accounting for the dip at $250 \mathrm{mg}$. As Grenni, Ancona, and Caracciolo suggested, there was a loss of biodiversity, which caused the increase in respiration.

Knowing this, the need for better management of antibiotics in agriculture is of utmost importance. The study has shown that we possibly do not know as much as we had thought about the ecological effect of antibiotics. With the possibility that they could have similar positive effects on growth and productivity as fertilizer pollution, the potential dangers increase. Steps need to be taken by those involved with the proper treatment of water and the prevention of agriculture pollution in order to stop further antibiotic pollution due to the greater mystery in its effects and thus the greater risk it poses. In addition, more information regarding antibiotics must be gathered to identify how these molecules can affect an aquatic ecosystem as a whole in order to have more information regarding the new issues that have arised from the results of this study.

With the potential risk of antibiotics proposed by the study, more research must be conducted. The first study that should be conducted is a repeat study of this one in order to verify the findings. This is a fundamental aspect to the scientific research process and must be conducted in order to have the results and conclusions be accepted by the scientific community. In this study in particular, due to the small scale of the research and the few trials conducted, a repeat study with more trials and rivers tested should be conducted for more conclusive results. In addition, a follow up should be done using different antibiotics of varying types to see how they would differ from the effects those tested in this study had in order to identify if that has a significant role in the change in respiration rate at various concentrations. Furthermore, research should be done on a microscale to identify the effects on specific organisms isolated from the water as other studies have done for different research. Since this study was a done looking at a sample with multiple types of unknown organisms, yielding a macroscale, seeing how antibiotics affect the specific organisms within the sample would all allow for a more definitive result to be gathered that would be less subject to possible errors that may have arose. Looking further into the subject of antibiotics concentration is important in understanding the impact our pollution has on the environment and to identify the actions needed to be taken to reduce their effects

\section{References}

Bashir, K. M. I., Cho, M. G. (2016). The effect of kanamycin and tetracycline on growth and photosynthetic activity of two chlorophyte algae. BioMed Research International. 2016. 1-8.

Calculate Correlation Co-efficient. n.d. Retrieved from https://www.calculators.org/math/correlation.php

Grenni P., Ancona V., Caracciolo A. B. (2018). Ecological effects of antibiotics on natural ecosystems: A review. Microchemical Journal. 25-39.

Kohanski M. A., Dwyer D. J., Collins J. J. (2010). How antibiotics kill bacteria: from targets to networks. Nat Rev Microbiol. 2010. 8(6). 423-435

Kolpin D., Furlong E., Meyer M., Thurman E. M., Zaugg S., Barber L., Buxton H. (2002). Pharmaceuticals, Hormones, and Other Organic Wastewater Contaminants in U.S. Streams, 1999-2000: A National Reconnaissance. USGS Staff. Published Research. 68.

Molot, L. A. (2017). The effectiveness of cyanobacteria nitrogen fixation: Review of bench top and pilot scale nitrogen removal studies and implications for nitrogen removal programs. Environmental Reviews, 25(3), 292-295.

Pick F. R. (2016). Blooming algae: a Canadian perspective on the rise of toxic cyanobacteria. Canadian Journal of Fisheries \& Aquatic Sciences. 73(7):1149-1158.

Onuoha, S. C. (2017). The Prevalence of Antibiotic Resistant Diarrhogenic Bacterial Species in Surface Waters, South Eastern Nigeria. Ethiopian Journal Of Health Sciences, 27(4), 319-330.

Ventola C. L. (2015). The Antibiotic Resistance Crisis. Pharmacy and Therapeutics. 40(4): 277-283.

Zhang, X., Li, Y., Liu, B., Wang, J., Feng, C., Gao, M., \& Wang, L. (2014). Prevalence of Veterinary Antibiotics and AntibioticResistant Escherichia coli in the Surface Water of a Livestock Production Region in Northern China. PLoS ONE, 9(11), 1-11. 